\title{
The 2008 Kavli Prize in Nanoscience: Carbon Nanotubes
}

\author{
Ali Javey* \\ Department of Electrical Engineering and Computer Sciences, University of California at Berkeley, Berkeley, California 94720, and Materials Sciences Division, Lawrence \\ Berkeley National Laboratory, Berkeley, California 94720
}

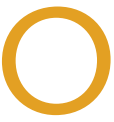

ver the years, the development of new materials with welldefined properties has been the enabling force for novel technological applications. The properties of materials are not only governed by their atomic compositions and chemical bonding, but also by their dimensions and length scales. Interesting properties arise when a material system approaches molecular dimensions. At such small scales, materials inherit some of the properties of molecules, resulting in unique optical, electrical, chemical, and mechanical characteristics and novel functionalities. This miniaturization trend was first envisioned by Richard Feynman in his talk, "There is Plenty of Room at the Bottom" at the 1959 annual meeting of the American Physical Society. Since then, a wide range of materials with reduced dimensions have been synthesized and characterized. ${ }^{1-7}$ One particularly interesting example of miniatur-

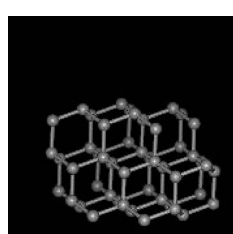

diamond

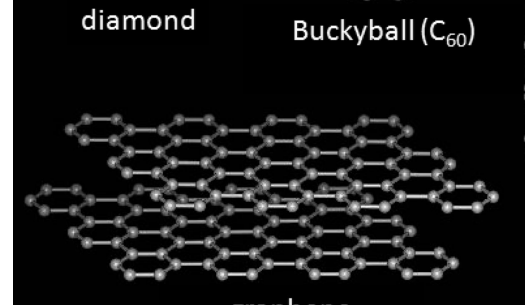

graphene

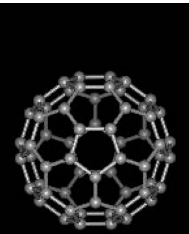

Buckyball $\left(\mathrm{C}_{60}\right)$

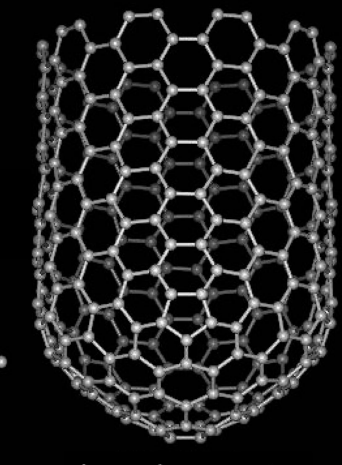

$(10,10)$ nanotube
Figure 1. Forms of crystalline carbon. A wide range of carbon structures with different physical, electrical, and mechanical properties are attainable. Reproduced with permission from ref 6 . Readers may view, browse, and/or download material for temporary copying purposes only, provided these uses are for noncommercial personal purposes. Except as provided by law, this material may not be further reproduced, distributed, transmitted, modified, adapted, performed, displayed, published, or sold in whole or part, without prior written permission from the American Physical Society. For information, see http://publish.aps.org/linkfaq.html.
ABSTRACT The 2008 Kavli Prize in Nanoscience was awarded to Dr. Sumio lijima for his hroterties. Great progress

ized structures are one-dimensional (1-D) nanomaterials, such as carbon nanotubes and nanowires (NWs), with molecular-scale diameters and microscale lengths. ${ }^{1-6,8-15}$ In recent years, significant progress has been made in the synthesis, assembly, underding of the fundamental properties, design of novel applications based on

Carbon Nanotubes. Carbon exhibits remarkable properties. There are multiple different forms of crystalline carbon with different bonding configurations and geometric dimensions. Carbon can be found in 3-D (diamond or graphite), 2-D (graphene sheets), 1-D (carbon nanotubes), or 0-D (buckyball) structures (Figure 1). ${ }^{6,8}$ Each of the above carbon materials exhibits distinct properties. For instance, diamond is insulating, graphite is semimetallic, and carbon nanotubes are uniquely metallic or semiconducting, depending on their structure. Carbon nanotubes are atomically well-defined, cylindrical structures that are hollow inside and
*Address correspondence to ajavey@eecs.berkeley.edu.

Published online July 22, 2008. $10.1021 / \mathrm{nn} 8003982$ CCC: $\$ 40.75$

(C) 2008 American Chemical Society 
Iijima was studying the

carbon materials formed

on the negative end of the

cathode electrode of an

arc-discharge evaporator

when he observed

MWNTs by transmission

electron microscopy.

Shortly thereafter, the

synthesis of SWNTs by

arc-discharge was

reported.

consist of either one (i.e., single-walled carbon nanotubes, SWNTs) or multiple (i.e., multiwalled carbon nanotubes, MWNTs) shells of carbon in a $\mathrm{sp}^{2}$ bonding configuration. ${ }^{15}$ With diameters as small as $\sim 0.4 \mathrm{~nm}(\sim 100000 \times$ thinner than a human hair) ${ }^{16,17}$ and lengths as long as $1 \mathrm{~cm}$, these high-aspect ratio materials are perhaps the closest reallife analogue to an ideal 1-D system (Figure 2). Their unique carbon-carbon bonding network and 1-D structure give rise to a number of interesting properties. For instance, nanotubes are among the strongest known materials, conduct electricity better than copper, and are efficient transporters of heat. $^{8-15}$ Depending on their chirality, nanotubes exhibit different electrical and optical properties. While this presents a singular opportunity for exploring novel concepts, it also presents a major challenge toward large-scale and reliable integration for certain applications that demand high monodispersity.

Discovery and Synthesis. Tube-like carbon nanostructures were first observed as early as $1952 .^{18,19}$ However, it was not until nearly four decades later when Dr. Sumio lijima reported the observation of MWNTs in the journal of Nature that created worldwide interest and excitement, and resulted in the development of an entirely new materials field. ${ }^{1}$ lijima was studying the carbon materials formed on the negative end of the cathode electrode of an arc-discharge evaporator when he observed MWNTs by transmission electron microscopy. Shortly thereafter, the synthesis of SWNTs by arc-discharge was reported. ${ }^{2,20}$ Since then, a number of other methods have been demonstrated for high-quality and high-yield synthesis of nanotubes, including laser ablation and chemical vapor deposition techniques. $^{21-23}$

Monodispersity. A wide range of selective synthesis, purification, separation, and destruction methods for attaining monodisperse SWNTs of well-defined chirality and diameter have been explored with varying degrees of success. $^{23-26}$ Selective synthesis is perhaps the most attractive approach for applications that require direct growth and positioning of nanotubes on substrates for largescale integration, either in vertical or horizontal configurations. Through trial and error, it has been found that growth conditions, including temperature, pressure, carbon source, plasma, and the metal catalyst material can all affect the overall distribution of nanotube chiralities and diameters grown. Of these parameters, tuning the catalyst material and the use of plasma-enhanced chemical vapor deposition seem to be the most promising for selective

syntheses. ${ }^{23,24}$ Despite these efforts, it is still challenging to synthesize nanotubes controllably of one chirality with $>90 \%$ purity. On the other hand, a number of solutionbased purification and separation methods for postsynthesis processing of nanotubes have been shown to enable better control of the dispersity of nanotubes. In particular, density gradient ultracentrifugation has enabled the separation of nanotubes with $>97 \%$ purity. ${ }^{25}$ Further improvement toward mono-dispersity may be attained by a combination of selective syntheses and postgrowth purification and separations. ${ }^{23}$

Properties and Applications. Due to their unique properties, many novel applications based on carbon nanotubes have been envisioned and demonstrated, including electronics, optoelectronics, sensors, drug delivery shuttles, composites, and more. ${ }^{8-15,27-43}$ Particularly, nanotube electronics have been the focus of a wide range of academic and industrial research efforts. ${ }^{9-11,13,27-33}$ The high electron and hole mobility $(\mu \approx$ $10000 \mathrm{~cm}^{2} \mathrm{~N} \cdot \mathrm{s}$ ) of nanotubes, their

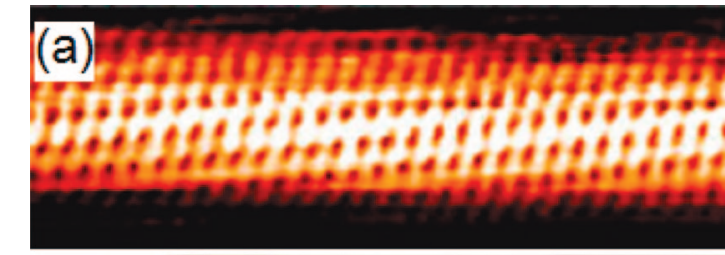

(b)
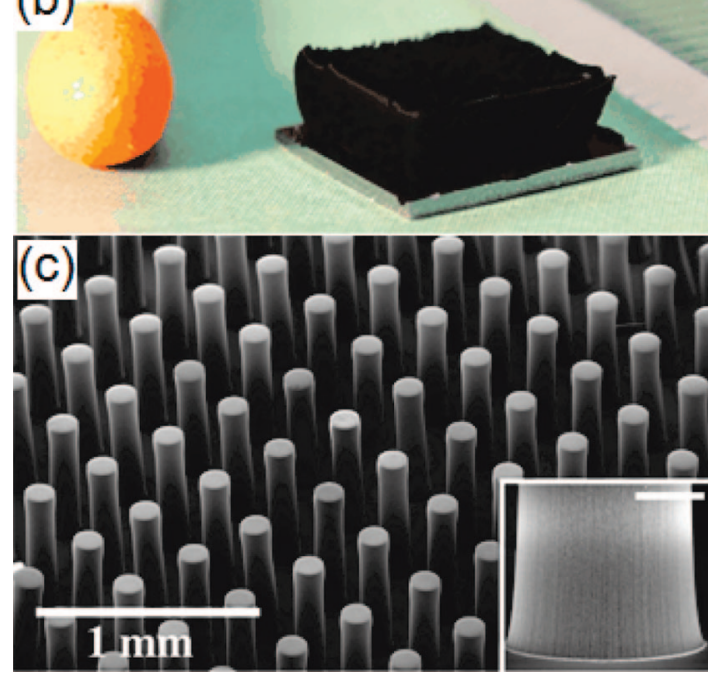

Figure 2. SWNT structures. (a) A scanning tunneling microscopy image, showing the atomic structure of a SWNT. Adapted with permission from ref 15. Copyright 1998 Macmillan Publishers Ltd. (b) Ultralong, high-yield and impurity-free synthesis of vertically aligned SWNTs on Si substrates is achieved by water-assisted chemical vapor deposition as first demonstrated by S. lijima and colleagues. A matchstick on the left is shown for size reference. (c) Scanning electron microscopy (SEM) images of patterned bundles of vertically aligned nanotubes grown by the same technique as in panel b. Inset scale bar is $\mathbf{5 0}$ $\mu \mathrm{m}$. Panels $b$ and $c$ are reproduced with permission from ref 22. Copyright 2004 American Association for the Advancement of Science. 
compatibility with high-к gate dielectrics without electron transport degradation, and ability to form metal ohmic contacts readily make these molecular structures an ideal channel material for high-performance nanoscale transistors. ${ }^{9,13,30-32}$ Furthermore, owing to their 1-D nature, better electrostatics can be attained by coaxial gating of nanotubes, thereby minimizing the various short-channel effects that are often encountered in nanoscale, planar devices. ${ }^{30}$ Ballistic nanotube transistors, free of any carrier scattering, have already been shown to be capable of delivering $\sim 30 \times$ higher ON current density (ON current is proportional to switching speed) as compared to stateof-the-art Si devices, therefore demonstrating the potential of using chemically synthesized molecular-scale structures for high-performance and low-power electronics (Figure 3). ${ }^{30}$ While the potential of nanotube electronics has been demonstrated, challenges in the assembly and purification of nanotubes need to be addressed to enable their integration for large-scale manufacturing. A number of approaches for achieving well-aligned nanotube arrays on substrates have been explored, including substrateinduced aligned growth and solutionbased Langmuir-Blodgett techniques. ${ }^{33}$ However, further improvements in the deterministic positioning of nanotubes with nanometer-scale accuracy over large areas are still needed. Another potential application of nanotubes is for the channel material of thinfilm transistors (TFTs), consisting of either parallel arrays or thin films of nanotubes. ${ }^{33}$ Unlike nanoelectronics, TFTs have a higher tolerance for misalignment and purity of SWNTs. Furthermore, because of their unique mechanical properties, nanotubes are highly flexible, making them ideal for integration in bendable, plastic electronics. Beside the integration of nanotubes for active device elements, they may also be utilized for high-performance interconnects.

The scaling of interconnects is perhaps one of the most significant challenges facing the continuation of the aggressive scaling of integrated circuits.
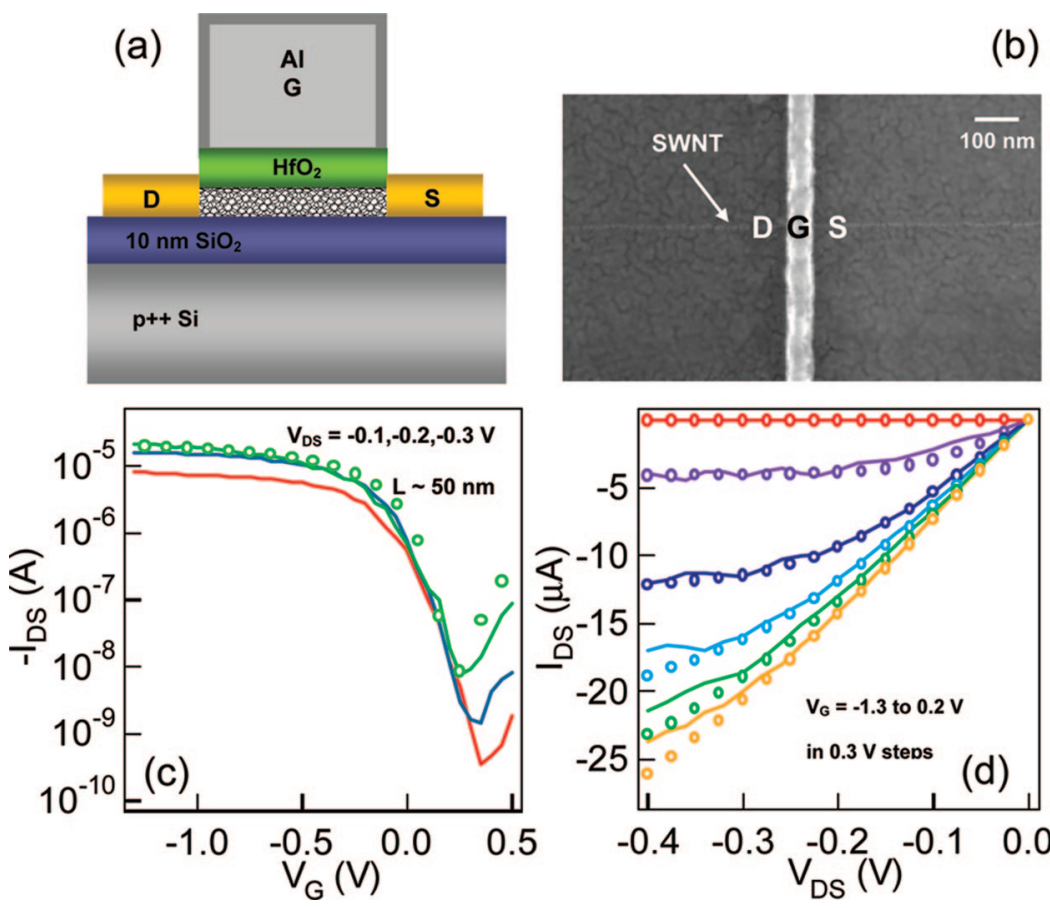

(b)

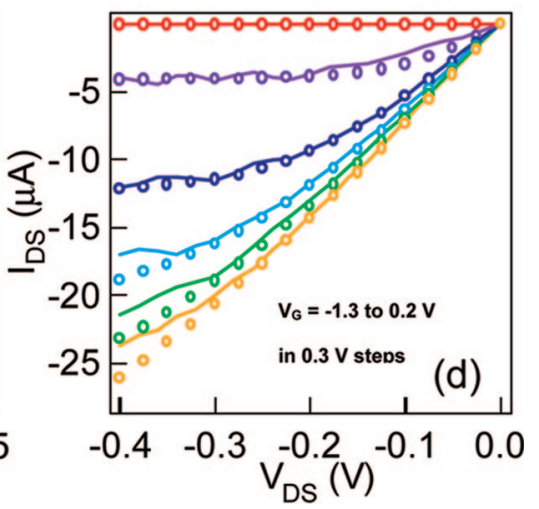

Figure 3. Self-aligned near-ballistic SWNT transistors. (a) Side-view schematic of a device. (b) SEM image showing the top-view of a device. The nanotube appears faint under the thin Pd electrodes. (c) Transfer characteristics for a nanotube device with length $L \approx 50 \mathrm{~nm}$ and diameter $d \approx 1.7 \mathrm{~nm}$ at different biases $\left(V_{\mathrm{DS}}\right)$. (d) Output characteristics of the same device. Solid lines are experimental data and symbols are ballistic quantum simulation, showing a good match between experiments and theory and suggesting near-ideal transport in the nanotube transistors. The transistors deliver $\sim 30 \times$ higher ON current density (normalized by $2 d$ ) at an operating voltage $V_{\mathrm{DD}}=0.3$ as compared to 90 -nm node Si technology devices. Reproduced with permission from ref 30. Copyright 2004 American Chemical Society.

At small scales, metal interconnects suffer from electromigration and high parasitic resistances. In contrast, nanotubes are immune to electromigration owing to their strong carbon-carbon $\mathrm{sp}^{2}$ network and exhibit lower resistances than a copper wire of similar dimensions. ${ }^{9-11,13,32}$ Additionally, nanotube films may be utilized as transparent conducting electrodes for certain applications, such as displays. Compared to the commonly used indium tin oxide electrodes, nanotube films show lower resistance for the same transparency level. ${ }^{34,35}$

Another interesting application of carbon nanotubes is in chemical and biological sensors. Since all of the carbon atoms are on the surface, SWNTs are highly sensitive to their environment. For in-
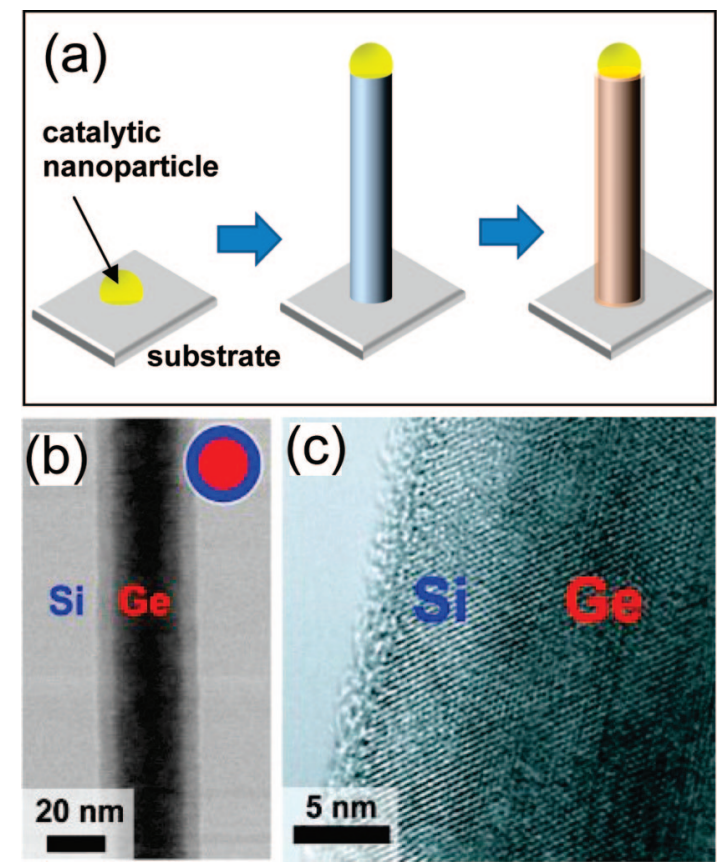

Figure 4. Core/shell nanowire heterostructures. (a) Schematic of a generic approach used to synthesize core/shell nanowires. In the first step, the nanowire core is grown followed by the deposition of the shell in a second step. $(b, c)$ Transmission electron microscopy images of Ge/Si core/shell nanowire heterostructures, showing abrupt junctions. Panels $b$ and $c$ reproduced with permission from ref 46. Copyright 2002 Macmillan Publishers Ltd. 


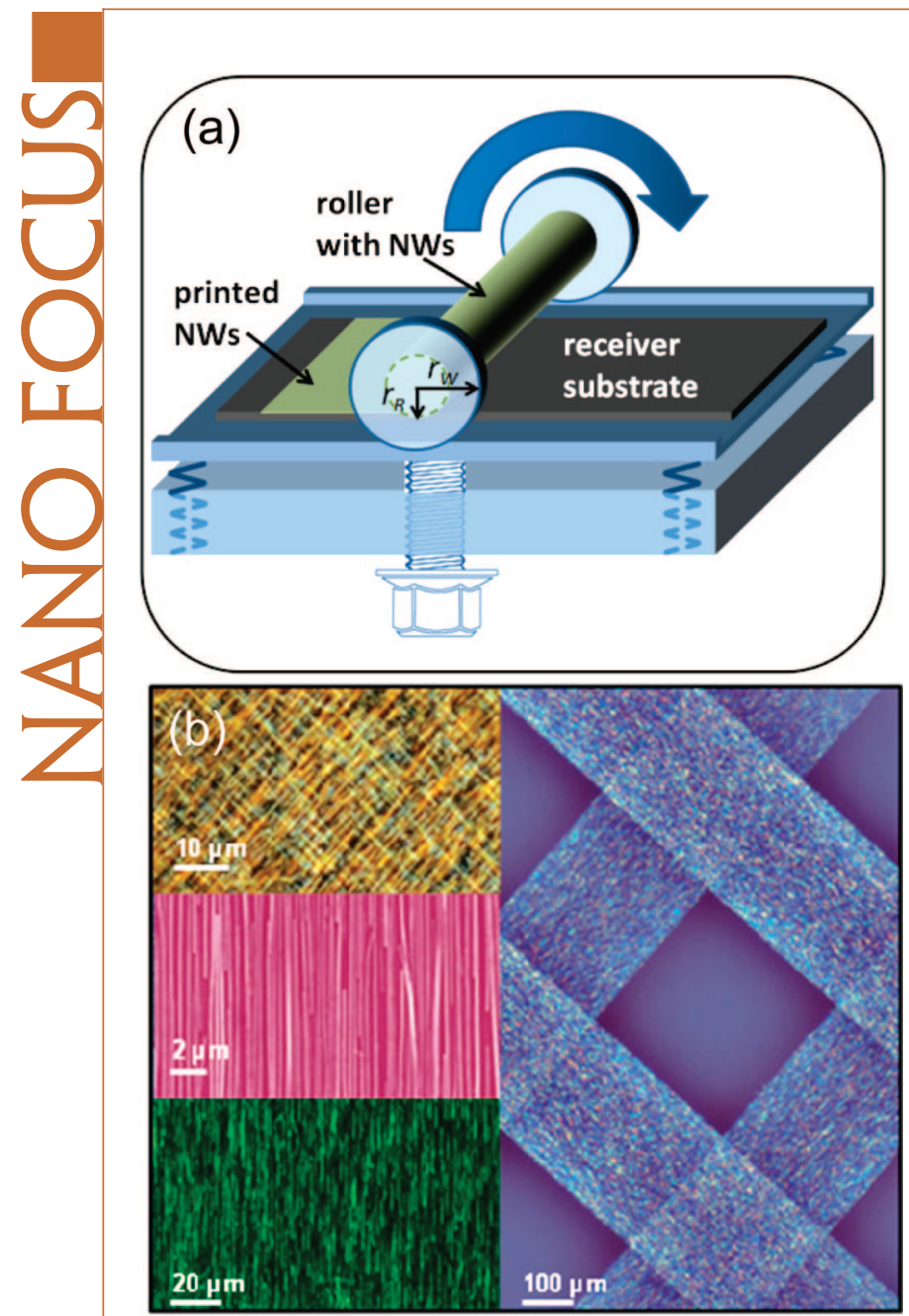

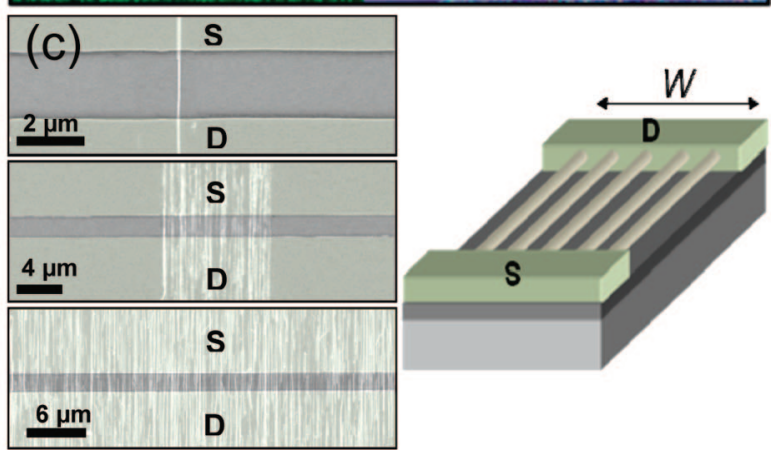

Figure 5. Printing of nanowires on substrates. (a) A schematic of a roll printing setup used for the assembly of parallel arrays of nanowires on substrates. Nanowires are first grown using chemical vapor deposition on a cylindrical glass substrate (i.e., roller), and are then transferred to a receiver substrate by a roll printing process. Adapted with permission from ref 57. Copyright 2007 American Institute of Physics. (b) Images of printed Ge and Si nanowire superstructures on $\mathrm{Si} / \mathrm{SiO}_{2}$ substrates. (c) The printed nanowire arrays can be configured as device elements. From top to bottom, SEM images of back-gated single NW FET, and 10 and $250 \mu \mathrm{m}$ wide parallel arrayed NW FETs. The effective channel width is defined by the number of nanowires and the pitch. Reproduced with permission from ref 56. Copyright 2008 American Chemical Society.

species and its absorption on the surface of SWNTs results in an effective "hole" doping of the devices by charge transfer, therefore modulating the conductance. Similar to other electronic nose sensors, carbon nanotube sensors suffer from inadequate specific response to various analytes. ${ }^{32}$ In the future, surface functionalization and/or large-scale multiplexing of nanotube sensors may be utilized to enable better specificity in the response and detection. ${ }^{36}$ Additionally, the large surface-areato-volume ratio of nanotubes presents an ideal opportunity for energy storage applications, including supercapacitors and fuel cells. $^{39,40}$

Nanotubes also exhibit superb mechanical properties. ${ }^{41-43}$ The carbon-carbon $\mathrm{sp}^{2}$ bonding is among the strongest known chemical bonds in nature. As a result, nanotube structures exhibit the highest tensile strength and elastic modulus of all materials. ${ }^{8,42}$ By utilizing their mechanical properties, researchers have demonstrated strong yet lightweight nanotube-based composites for a number of applications, ${ }^{43}$ some of which have already been commercialized.

stance, nanotube-based electronic sensors with 100 part-per-trillion sensitivity to $\mathrm{NO}_{2}$ have been demonstrated. ${ }^{36} \mathrm{NO}_{2}$ is a strong electron-withdrawing gas
Finally, nanotubes can be readily functionalized for biomedical applications. ${ }^{38}$ Although the exact biological toxicity ${ }^{37}$ of nanotubes remains a controversial topic, depending on their surface functionalization and purity, SWNTs have been shown to be compatible with certain cellular systems as their molecularscale dimensions allow them to penetrate into the desired cells for drug delivery or bioimaging functionalities.

The potential applications listed here present only a fraction of those that have been proposed and envisioned, which attests to the versatility of nanotubes as functional nanomaterials.

Crystalline, Inorganic Nanowires. Besides carbon nanotubes, inorganic NWs present another unique class of 1-D nanomaterials with distinct chemical, physical, and mechanical properties. ${ }^{4,5}$ NWs are single crystalline, cylindrical materials with tunable diameters $(d \geq$ $2 \mathrm{~nm}$ ), lengths, and elemental composition. ${ }^{4}$ Unlike carbon nanotubes, most inorganic NWs do not exhibit a band-gap dependence on their "chirality" (i.e., crystal orientation), although the band gap does depend on NW diameter, especially for small diameter NWs, due to quantization effects.

The first synthesis of singlecrystalline NWs was reported in 1998 by Charles Lieber and colleagues in which a laser ablation method was used to synthesize Si NWs by the vapor-liquid-solid process. ${ }^{44}$ Since then, Lieber and others have demonstrated the syntheses of a wide range of NW materials, including heterostructures (Figure 4$)^{45-47}$ with advanced and well-defined functionalities and properties while controllably assembling them into hierarchical structures (Figure 5) and configuring them for a wide range of applications. ${ }^{48-66}$

Properties and Applications. While the material properties of carbon nanotubes are primarily governed by their chirality (and thus, their diameter), for NWs, the properties are tuned by both the diameter and the elemental composition, which adds another handle in controlling functionality. Over the past decade, NWs have been demonstrated as building blocks for a number of applications, including electronics, electro-optics, sensors, energy generation and storage, neural-interfaces, and more. ${ }^{45-66}$ 

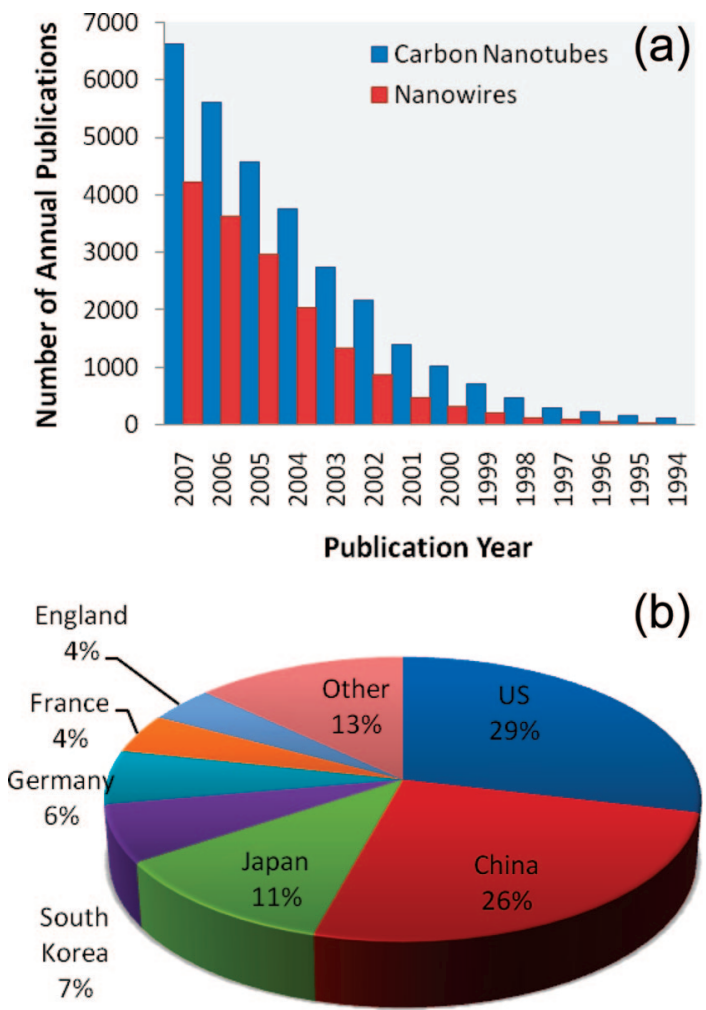

Figure 6. Carbon nanotube and nanowire journal publications. (a) Number of annual journal publications for 1994-2007, showing an astonishing growth in the field for this period. (b) Distribution of nanotube and nanowire publications by country of authorship. The data is obtained from the ISI Web of Science database (June 23, 2008).

To date, NWs with carrier mobilities as high as those of SWNTs for the same diameters $(d<5 \mathrm{~nm}$ ) have not yet been achieved, and it remains an open question as to whether such high mobilities (on the order of $10000 \mathrm{~cm}^{2} / \mathrm{V} \cdot \mathrm{s}$ ) will ever be attained in sub-5-nm NWs due to surface scattering and quantization effects. However, high-mobility $p$-type $\left(\mu_{\mathrm{h}} \approx 750 \mathrm{~cm}^{2} /(\mathrm{V} \cdot \mathrm{s})\right)$ and $n$-type $\left(\mu_{\mathrm{n}} \approx\right.$ $\left.10000 \mathrm{~cm}^{2} / \mathrm{V} \cdot \mathrm{s}\right)$ transistors based on $\mathrm{Ge} / \mathrm{Si}$ core/shell and InAs NWs have been demonstrated for diameters of $10-25 \mathrm{~nm} .{ }^{49,50,52}$ While there is no clear evidence for increased carrier mobilities over their bulk counterparts, the reduced dimensions of NWs result in improved gate coupling and reduced short channel effects, which are critical for achieving high performance in nanoscale devices. Additionally, NW heterostructures can be readily synthesized with controlled dimensions and elemental composition for enhanced transport properties as has been shown in the Ge/Si and InAs/InP core/shell NW structures. ${ }^{49,50}$ Another unique advantage of NWs for electronics is the ability either to grow NWs directly on Si substrates or first to grow and then to transfer them to receiver substrates for heterogeneous integration, ${ }^{53}$ without encountering the lattice mismatch difficulties often faced for planar films. Furthermore, the low processing temperatures for crystalline NWs enable multilayer stacking of high-performance electronic and sensor elements for multifunctional, 3-D integration. ${ }^{54,55}$ Interestingly, NWs can be assembled as highly ordered arrays by contact printing on unconventional substrates, such as plastic, glass, or paper for high-performance, "printable" macroelectronics (Figure 5). ${ }^{56,57}$

Semiconductor NWs have also been utilized for harvesting solar, mechanical, and thermal energies. ${ }^{60,64-66}$ For instance, allinorganic and inorganic-organic hybrid solar cells based on individual NWs and NW arrays have been demonstrated as possible routes toward cheap and yet reasonably efficient photovoltaics. $^{60,64,66}$ In another interesting approach, the piezoelectric properties of ZnO NWs were utilized to harvest the vibrational energy of the system for energy-generating fabrics. ${ }^{65}$ Optically active NWs have also been utilized for a variety of optoelectronic applications, including efficient lasers, light-emitting diodes, and highly sensitive, polarized photodetectors. ${ }^{58,59}$

Due to their nanoscale diameters, NW devices may be utilized for effective electrical interfacing and communication with neurons and other cellular systems. ${ }^{62}$ In this approach, nanoscale junctions have been formed between neurons and the NWs and used for spa- tially resolved and sensitive detection, stimulation, and inhibition of neuronal signal propagation along axons and dendrites ex vivo. Such a novel system may lead to better fundamental understanding of neuronal operation and communication while presenting a novel path toward future bioinspired electronics. Additionally, as for carbon nanotubes, the large surface-area-tovolume ratios of NWs makes them ideal materials for sensing applications. Welldefined surface functionalization approaches have been developed to enable highly sensitive and specific detection of various biological materials, including proteins and viruses. ${ }^{61}$ Such electronic biosensors may provide an efficient and economical detection method for early disease diagnoses.

\section{CONCLUSIONS AND PROSPECTS}

Since the early 1990s, the field of 1-D nanostructures has seen explosive progress in the controlled synthesis and detailed characterization of carbon nanotubes and NWs. These miniaturized materials have served as model systems for understanding basic phenomena in 1-D. Furthermore, the unique properties of nanotubes and NWs have led to the development of a wide range of technological applications. Undoubtedly, this exciting field will continue to grow and expand (Figure 6) with researchers ac-

Over the past decade,

inorganic nanowires have

been demonstrated as

building blocks for a

number of applications,

including electronics,

electro-optics, sensors,

energy generation and

storage, neural-interfaces,

and more. 
tively tackling the various processing challenges, including purification and assembly, while creating, developing, and exploiting new applications.

Acknowledgment. The Table of Contents graphic was reproduced from ref 13. Copyright 2006 World Scientific.

\section{REFERENCES AND NOTES}

1. Ijima, S. Helical Microtubules of Graphite Carbon. Nature 1991, 354, 56-58.

2. Ijima, S.; Ichihashi, T. Single-Shell Carbon Nanotubes of 1-nm Diameter. Nature 1993, 363, 603-605.

3. lijima, S.; Ichihashi, T.; Ando, Y Pentagons, Heptagons and Negative Curvature in Graphite Microtubule Growth. Nature 1992, 356, 776-778.

4. Lieber, C. M.; Wang, Z. L. Functional Nanowires. MRS Bull. 2007, 32, 99-104.

5. Lieber, C. M. The Incredible Shrinking Circuit. Sci. Am. Special Ed. 2007, 17, 6471.

6. Smalley, R. E. Discovering the Fullerenes. Rev. Mod. Phys. 1997, 69, 723-730.

7. Alivisatos, A. P. Nanocrystals as a New Class of Macromolecules. In ISAC XXII International Congress Abstract Listings; Cytometry Part A; ISAC: Bethesda, MD, 2004; Vol. 59A, p 29.

8. Jorio, A., Dresselhaus, G., Dresselhaus, M. S., Eds.; Carbon Nanotubes; Springer: Berlin, 2008; Vol. 111.

9. Avouris, P.; Chen, J. Nanotube Electronics and Optoelectronics. Mater. Today 2006, 9 (10), 46-54.

10. McEuen, P. L; Fuhrer, M. S.; Park, H. K. Single-Walled Carbon Nanotube Electronics. IEEE Trans. Nanotechnol. 2002, 1, 78-85.

11. Dekker, C. Carbon Nanotubes as Molecular Quantum Wires. Phys. Today 1999, 52, 22-28.

12. Rogers, J. A. Slice and Dice, Peel and Stick: Emerging Methods for Nanostructure Fabrication. ACS Nano 2007, 1, 151-153.

13. Dai, H.; Javey, A.; Pop, E.; Mann, D.; Kim, Y.; Lu, Y. Electrical Transport Properties and Field-Effect Transistors of Carbon Nanotubes. NANO 2006, 1, 1-13.

14. Baughman, R. H.; Zakhidov, A. A.; de Heer, W. A. Carbon Nanotubes-the Route Toward Applications. Science 2002, 297, 787-792.

15. Wildoer, J. W. G.; Venema, L. C.; Rinzler, A. G.; Smalley, R. E.; Dekker, C. Electronic Structure of Atomically Resolved Carbon Nanotubes. Nature 1998, 391, 59-62.

16. Guan, L.; Suenaga, K.; lijima, S. Smallest Carbon Nanotube Assigned with Atomic Resolution Accuracy. Nano Lett. 2008, 8, 1530-6984.

17. Ajayan, P. M.; lijima, S. Smallest Carbon Nanotube. Nature 1992, 358, 23.

18. Radushkevich, L. V.; Lukyanovich, V. M. O Strukture Ugleroda, Obrazujucegosja Pri Termiceskom Razlozenii Okisi Ugleroda na Zeleznom Kontakte. Zurn. Fisic. Chim. 1952, 26, 88-95.
19. Oberlin, A.; Endo, M.; Koyama, T. Filamentous Growth of Carbon through Benzene Decomposition. J. Cryst. Growth 1976, 32, 335-349.

20. Bethune, D. S.; Klang, C. H.; de Vries, M. S.; Gorman, G.; Savoy, R.; Vazquez, J.; Beyers, R. Cobalt Catalyzed Growth of Carbon Nanotubes with Single Atomic Layer Walls. Nature 1993, 363, 605-607.

21. Kong, J.; Soh, H. T.; Cassell, A. M.; Quate, C. F.; Dai, H. Synthesis of Individual Single-Walled Carbon Nanotubes on Patterned Silicon Wafers. Nature 1998, 395, 878-881.

22. Hata, K; Futaba, D. N.; Mizuno, K.; Namai, T.; Yumura, M.; lijima, S. Water-Assisted Highly Efficient Synthesis of ImpurityFree Single-Walled Carbon Nanotubes. Science 2004, 306, 1362-1364.

23. Li, X.; Tu, X.; Zaric, S.; Welsher, K.; Seo, W. S.; Zhao, W.; Dai, H. Selective Synthesis Combined with Chemical Separation of Single-Walled Carbon Nanotubes for Chirality Selection. J. Am. Chem. Soc. 2007, 129, 15770-15771.

24. Li, Y.; Mann, D.; Rolandi, M.; Kim, W.; Ural, A.; Hung, S.; Javey, A.; Cao, J.; Wang, D.; Yenilmez, E.; et al. Preferential Growth of Semiconducting Single-Walled Carbon Nanotubes by a Plasma Enhanced CVD Method. Nano Lett. 2004, 4, 317-321.

25. Arnold, M. S.; Green, A. A.; Hulvat, J. F.; Stupp, S. I.; Hersam, M. C. Sorting Carbon Nanotubes by Electronic Structure via Density Differentiation. Nat. Nanotechnol. 2006, 1, 60-65.

26. Collins, P. C.; Arnold, M. S. Avouris, Ph. Engineering Carbon Nanotubes and Nanotube Circuits using Electrical Breakdown. Science 2001, 292, 706-709.

27. Tans, S. J.; Verschueren, A. R. M.; Dekker, C. Room-Temperature Transistor Based on a Single Carbon Nanotube. Nature 1998, 393, 49-52.

28. Martel, R.; Schmidt, T.; Shea, H. R.; Hertel, T. Avouris, Ph. Single- and Multi-Wall Carbon Nanotube Field-Effect Transistors. Appl. Phys. Lett. 1998, 73, 2447-2449.

29. Soh, H. T.; Morpurgo, A.; Kong, J.; Marcus, C.; Quate, C.; Dai, H. Integrated Nanotube Circuits: Controlled Growth and Ohmic Contacts to Single-Walled Carbon Nanotubes. Appl. Phys. Lett. 1999, 75, 627-629.

30. Javey, A.; Guo, J.; Farmer, D. B.; Wang, Q.; Yenilmez, E.; Gordon, R. G.; Lundstrom, M.; Dai, H. Self-Aligned Ballistic Molecular Transistors and Electrically Parallel Nanotube Arrays. Nano Lett. 2004, 4, 1319-1322.

31. Javey, A.; Guo, J.; Wang, Q.; Lundstrom, M.; Dai, H. Ballistic Carbon Nanotube Field-Effect Transistors. Nature 2003, 424, 654-657.

32. Javey, A.; Kong, J., Eds.; Carbon Nanotube Electronics; Springer: New York, in press.

33. Kang, S. J.; Kocabas, C.; Ozel, T.; Shim, M.; Pimparkar, N.; Alam, M. A.; Rotkin, S. V.; Rogers, J. A. High-Performance Electronics using Dense, Perfectly Aligned Arrays of Single-Walled Carbon Nanotubes. Nat. Nanotechnol. 2007, 2, 230-236.
34. Wu, Z.; Chen, Z.; Du, X.; Logan, J. M.; Sippel, J.; Nikolou, M.; Kamaras, K.; Reynolds, J. R.; Tanner, D. B.; Hebard, A. F.; et al Transparent, Conductive Carbon Nanotube Films. Science 2004, 305, 1273-1276.

35. Blackburn, J. L.; Barnes, T. M.; Beard, M. C.; Kim, Y.-H.; Tenent, R. C.; McDonald, T. J.; To, B.; Coutts, T. J.; Heben, M. J. Transparent Conductive Single-Walled Carbon Nanotube Networks with Precisely Tunable Ratios of Semiconducting and Metallic Nanotubes. ACS Nano 2008, 2, 1266-1274.

36. Qi, P.; Vermesh, O.; Grecu, M.; Javey, A.; Wang, Q.; Dai, H. Toward Large Arrays of Multiplex Functionalized Carbon Nanotube Sensors for Highly Sensitive and Selective Molecular Detection. Nano Lett. 2003, 3, 347-351.

37. Miyawaki, J.; Yudasaka, M.; Azami, T.; Kubo, Y.; lijima, S. Toxicity of SingleWalled Carbon Nanohorns. ACS Nano 2008, 2, 213-226.

38. Liu, Z.; Sun, X.; Nakayama-Ratchford, N.; Dai, H. Supramolecular Chemistry on Water-Soluble Carbon Nanotubes for Drug Loading and Delivery. ACS Nano 2007, 1, 50-56.

39. Kongkanand, A.; Kamat, P. V. Electron Storage in Single Wall Carbon Nanotubes. Fermi Level Equilibration in Semiconductor-SWCNT Suspensions. ACS Nano 2007, 1, 13-21.

40. Pushparaj, V. L.; Shaijumon, M. M.; Kumar, A.; Murugesan, S.; Ci, L.; Vajtai, R.; Linhardt, R. J.; Nalamasu, O.; Ajayan, P. M. Flexible Energy Storage Devices Based on Nanocomposite Paper. Proc. Nat. Acad. Sci. U.S.A. 2007, 104, 13574-13577.

41. Fennimore, A. M.; Yuzvinsky, T. D.; Han, W.-Q.; Fuhrer, M. S.; Cumings, J.; Zettl, A. Rotational Actuators Based on Carbon Nanotubes. Nature 2003, 424, 408-410.

42. Treacy, M. M. J.; Ebbesen, T. W.; Gibson, J. M. Exceptionally High Young's Modulus Observed for Individual Carbon Nanotubes. Nature 1996, 381, 678-680.

43. Ajayan, P. M.; Tour, J. M. Nanotube Composites. Nature 2007, 447, 1066-1068.

44. Morales, A. M.; Lieber, C. M. A Laser Ablation Method for the Synthesis of Crystalline Semiconductor Nanowires. Science 1998, 279, 208-211.

45. Wu, Y.; Fan, R.; Yang, P. Block-by-Block Growth of Si/SiGe Superlattice Nanowires. Nano Lett. 2002, 2, 83-86.

46. Lauhon, L. J; Gudiksen, M. S.; Wang, D.; Lieber, C. M. Epitaxial Core-Shell and Core-Multi-Shell Nanowire Heterostructures. Nature 2002, 420, 5761.

47. Björk, M. T.; Ohlsson, B. J.; Sass, T.; Persson, A. I.; Thelander, C.; Magnusson, M. H.; Deppert, K.; Wallenberg, L. R.; Samuelson, L. One-Dimensional Steeplechase for Electrons Realized. Nano Lett. 2002, 2, 87-89.

48. Huang, Y.; Duan, X.; Wei, Q.; Lieber, C. M. Directed Assembly of OneDimensional Nanostructures into 
Functional Networks. Science 2001, 291, 630-633.

49. Xiang, J.; Lu, W.; Hu, Y.; Wu, Y.; Yan, H.; Lieber, C. M. Ge/Si Nanowire Heterostructures as High-Performance Field-Effect Transistors. Nature 2006, 441, 489-493.

50. Jiang, X.; Xiong, Q.; Nam, S.; Qian, F.; Li, Y.; Lieber, C. M. InAs/InP Radial Nanowire Heterostructures as High Electron Mobility Devices. Nano Lett. 2007, 7, 3214-3218.

51. Chung, S.-W.; Yu, J.-Y.; Heath, J. R. Silicon Nanowire Devices. Appl. Phys. Lett. 2000, 76, 2068-2070.

52. Bryllert, T.; Wernersson, L.-E.; Fröberg, L. E.; Samuelson, L. Vertical HighMobility Wrap-Gated InAs Nanowire Transistor. IEEE Electron Device Lett. 2006, 27, 323-325.

53. Fan, Z.; Ho, J. C.; Jacobson, Z. A.; Razavi, $\mathrm{H}$.; Javey, A. Large Scale, Heterogeneous Integration of Nanowire Arrays for Image Sensor Circuitry. Proc. Natl. Acad. Sci. U.S.A., in press.

54. Javey, A.; Nam, S.; Friedman, R. S.; Yan, H.; Lieber, C. M. Layer-by-Layer Assembly of Nanowires for Three-Dimensional, Multifunctional Electronics. Nano Lett. 2007, 7, 773-777.

55. Ahn, J-H.; Kim, H-S.; Lee, K. J.; Jeon, S.; Kang, S. J.; Sun, Y.; Nuzzo, R. G.; Rogers, J. A. Heterogeneous Three-Dimensional Electronics by Use of Printed Semiconductor Nanomaterials. Science 2006, 314, 1754-1757.

56. Fan, Z.; Ho, J. C.; Jacobson, Z. A.; Yerushalmi, R.; Alley, R. L.; Razavi, H.; Javey, A. Wafer-Scale Assembly of Highly Ordered Semiconductor Nanowire Arrays by Contact Printing. Nano Lett. 2008, 8, 20-25.

57. Yerushalmi, R.; Jacobson, Z. A.; Ho, J. C.; Fan, Z.; Javey, A. Large Scale, Highly Ordered Assembly of Nanowire Parallel Arrays by Differential Roll Printing. Appl. Phys. Lett. 2007, 91, 203104-1203104-4.

58. Wang, J.; Gudiksen, M. S.; Duan, X.; Cui, Y.; Lieber, C. M. Highly Polarized Photoluminescence and Photodetection from Single Indium Phosphide Nanowires. Science 2001, 293, 1455-1457.

59. Duan, X.; Huang, Y.; Cui, Y.; Wang, J.; Lieber, C. M. Indium Phosphide Nanowires as Building Blocks for Nanoscale Electronic and Optoelectronic Devices. Nature 2001, 409, 66-69.

60. Tian, B.; Zheng, X.; Kempa, T. J.; Fang, Y.; Yu, N.; Yu, G.; Huang, J.; Lieber, C. M. Coaxial Silicon Nanowires as Solar Cells and Nanoelectronic Power Sources. Nature 2007, 449, 885-889.

61. Patolsky, F.; Zheng, G.; Lieber, C. M. Fabrication of Silicon Nanowire Devices for Ultrasensitive, Label-Free, Real-Time Detection of Biological and Chemical Species. Nat. Protocols 2006, 1, 1711-1724.

62. Patolsky, F.; Timko, B. P.; Yu, G.; Fang, Y.; Greytak, A. B.; Zheng, G.; Lieber, C. M. Detection, Stimulation, and Inhibition of Neuronal Signals with High-Density
Nanowire Transistor Arrays. Science 2006, 313, 1100-1104.

63. Huang, M. H.; Mao, S.; Feick, H.; Yan, H.; Wu, Y.; Kind, H.; Weber, E.; Russo, R.; Yang, P. Room-Temperature Ultraviolet Nanowire Nanolasers. Science 2001, 292, 1897-1899.

64. Lewis, N. S. Toward Cost-Effective Solar Energy Use. Science 2007, 315, 798-801.

65. Wang, X.; Song, J.; Liu, J.; Wang, Z. L. Direct-Current Nanogenerator Driven by Ultrasonic Waves. Science 2007, 316, 102-105.

66. Law, M.; Greene, L. E.; Johnson, J. C.; Saykally, R.; Yang, P. Nanowire DyeSensitized Solar Cells. Nat. Mater. 2005, 4, 455-459. 